\title{
Interest of using a micro-meter spatial resolution to study SiC semi- conductor devices by Optical Beam Induced Current (OBIC)
}

\author{
Camille SONNEVILLE ${ }^{1) a}$, Dominique PLANSON ${ }^{1 \text { b) }}{ }^{*}$, Luong Viet PHUNG ${ }^{1)}$, Pascal BEVILACQUA ${ }^{1)}$, \\ Besar ASLLANI ${ }^{12)}$ \\ 1) Univ Lyon, INSA Lyon, Ecole Centrale de Lyon, Université Claude Bernard Lyon 1, \\ CNRS, Ampère, F-69621, Villeurbanne, France \\ 2) SuperGrid Institute, 23 rue Cyprian CS 50289, 69628 Villeurbanne Cedex-France \\ a) camille.sonneville@insa-lyon.fr \\ b) dominique.planson@insa-lyon.fr
}

Keywords: OBIC characterization, Silicon Carbide High Voltage Diodes, Wide Band Gap semiconductor, TCAD

\begin{abstract}
In this paper we present a new test bench called micro-OBIC used to characterized wide band gap semi-conductor. Micro-OBIC allows to measure an Optical Beam Induced Current (OBIC) signal with a microscopic spatial resolution. We used micro-OBIC to characterize peripheral protection such as MESA, JTE or JTE rings in high voltage $\mathrm{SiC}$ device.
\end{abstract}

\section{Introduction}

High voltage devices, up to $10 \mathrm{kV}$, are mandatory for high power converters as they will be used in HVDC energy transmission network in the future [1]. For the sake of power losses, bipolar devices are interesting due to the conductivity modulation of the thick and low doped layer. Efficient peripheral protections are requested to avoid electric field crowding close to the edges of the device. Thus, non-destructive characterization techniques are useful to study these features.

UV Laser is used to generate electron-hole pairs into Wide Band-Gap (WBG) semiconductors (SiC, $\mathrm{GaN}$ or $\mathrm{Ga}_{2} \mathrm{O}_{3}$ ). In the space charge region (SCR), the electric field drives the collected carriers and a current, named Optical Beam induced Current (OBIC), can be measured. This induced current is then directly related to the electrical field in the device. OBIC is a non-destructive technique, which has been previously successfully used to characterize High Voltage (HV) SiC devices [2,3,4,5]. In order to fully benefit of the advantages provided by WBG semiconductors materials and to avoid premature breakdown of the high voltage devices, it is mandatory to have efficient peripheral protections such as a combination of MESA with JTE or MESA with JTE and JTE rings as described in [6]. The OBIC characterization can help Technology Computer-Aided Design (TCAD) and the device process to optimize the efficiency of the peripheral protections by analyzing the electric field distribution in the structure and especially at the junction periphery. In this paper, we will present an in-house testbench called micro-OBIC which will allow us to characterize HV PiN diodes with a micro-meter spatial resolution. Theoretically, a small spot size should allow the accurate determination of the OBIC signal at specific location such as MESA, JTE and JTE rings.

\section{Experimental and set-up}

\section{Description of the micro-OBIC test bench}

We developed in Ampere laboratory a custom OBIC test-bench called micro-OBIC. A laser emitting UV is used to generate electron-hole pairs into WBG semiconductors. The laser is a Nd:YLF pulsed laser from spectra physics emitting a gaussian beam at $349 \mathrm{~nm}$ with a mean power around $4.10^{-3}$ Watt. The original beam exiting the laser has a waist diameter around $170 \mu \mathrm{m}$. In order to reduce the laser beam spot size, we developed a testbench represented in figure 1. The laser beam power is first controlled by a set of neutral density filter, before passing through a X10 beam expander. Finally, the 
laser spot is focused by a long-distance X15 Thorlabs microscope objective. A beam expander allows to illuminate a wider part of the microscope objective aperture, in order to reduce diffraction and then optimize the final laser spot size. To estimate the final diameter of the gaussian beam, we use the following equation [7]:

$$
\omega^{\prime}=\frac{\lambda f^{\prime}}{10 \omega \pi}
$$

Where $\omega^{\prime}$ is the final waist diameter, $\lambda$ is the laser wavelength $(349 \mathrm{~nm}), \mathrm{f}^{\prime}$ is the objective equivalent focal length $(13 \mathrm{~mm})$ and $\omega$ is the original laser beam waist diameter $(170 \mu \mathrm{m})$. Thanks to Eq. 1, we estimated $\omega^{\prime}$ and thus the spot diameter around $1 \mu \mathrm{m}$. The $(\mathrm{x}, \mathrm{y})$ spatial resolution of this new OBIC test-bench is estimated around $1 \mu \mathrm{m}$. The $\mathrm{z}$ focus was manually realized by optimizing the OBIC signal in the $\mathrm{p}^{+}$region (top anode level). Moreover, the focus depth is greater than the mesa height so there is no need to readjust the focus on the JTE level.

The HV device under test (DUT) is fixed on a X, Y, Z motorized stage. The motorized stage, controlled by a homemade Labview program, allows us to move the DUT under the laser beam focus point. Induced current is then measured with a Keithley 237 High-Voltage Source-Measure Unit (SMU). The DUT can be reverse biased by applying voltage thanks to the SMU (source measure unit). The testbench allows us to realize a spatial mapping of OBIC signal, either on a $(\mathrm{X}, \mathrm{Y})$ plane or only on X (or Y) lines. For X or Y lines, the step between two displacements of the laser beam is $0.25 \mu \mathrm{m}$. For $(\mathrm{X}, \mathrm{Y})$ plane, the step displacement of the laser beam in both directions is $3 \mu \mathrm{m}$. Fig. 1 represents schematically the micro-OBIC test-bench.

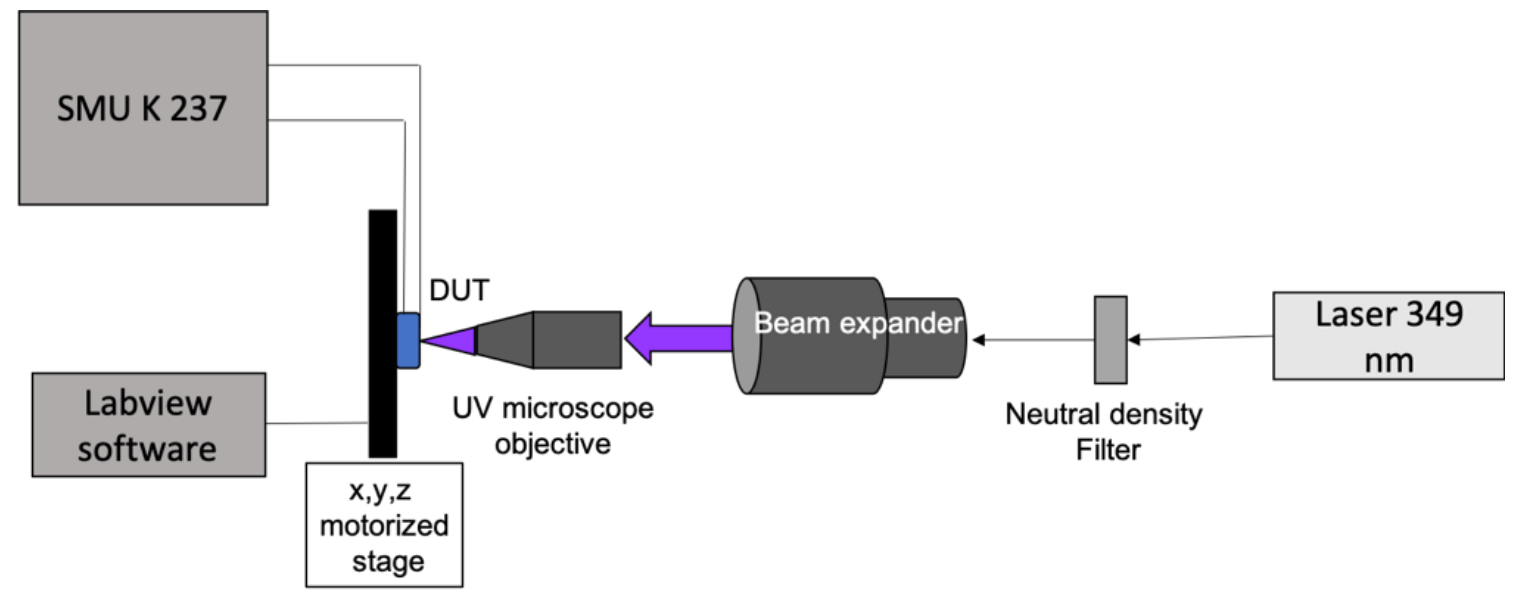

Fig. 1 Schematic representation of the micro-OBIC testbench

\section{Diode description}

Experimental micro-OBIC scans were performed on high voltage PiN diode (10 kV-class). 10KVclass PiN diode means that these diodes were designed by TCAD in collaboration with CALY Technologies to sustain up to $10 \mathrm{kV}$ when reverse biased. However, due to technological issues during ion implantation of the JTE, the best sustainable breakdown voltage is around $9 \mathrm{kV}$. In the frame of this paper, we used "test" diodes, i.e without polyimide passivation. These "test" diodes exhibit a lower breakdown voltage.

The high voltage PiN diodes were fabricated on a $4 \mathrm{H}-\mathrm{SiC}$ wafer using a $110 \mu \mathrm{m}$ thick epilayer with a doping concentration of $7.10^{14} \mathrm{~cm}^{-3}$. These diodes are protected by a combination of a MESA and a JTE of $400 \mu \mathrm{m}$ length (p doping concentration of $7.10^{16} \mathrm{~cm}^{-3}$, thickness $0.7 \mu \mathrm{m}$ ) and have whether (diode 2) or not 8 JTE rings (diode 1), as shown in Fig.2a) and b).

The diodes fabrication was led by CALY Technologies on a 4 inches wafer and realized in the cleanroom of the IBS company. Square shape diodes with a bend radius of $600 \mu \mathrm{m}$ have been implemented with different edge terminations. An elementary cell view of diode mask is shown in 
Fig.3. Polyimide secondary passivation has been removed in this field. There is still a thin $\mathrm{SiO}_{2}$ layers as primary passivation, which is transparent to UV-light. The breakdown voltage has been measured in a vacuum chamber at ISL [8] for both diodes (diode 1: $\mathrm{V}_{\mathrm{br}}=5743 \mathrm{~V}$, diode 2 : $\mathrm{V}_{\mathrm{br}}=5578 \mathrm{~V}$ ).
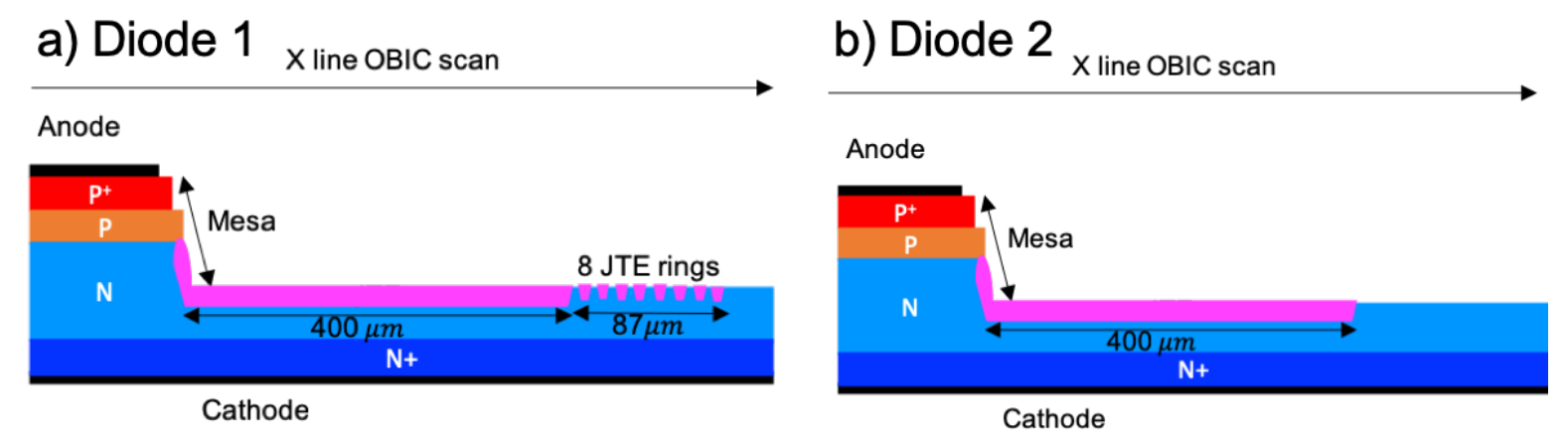

Fig. 2a) (left) Structure of the 8 JTE rings HV PiN diodes (diode 1), Fig. 2b) (right) Structure of the JTE without ring HV PiN diodes (diode 2). Schema are not to scale.

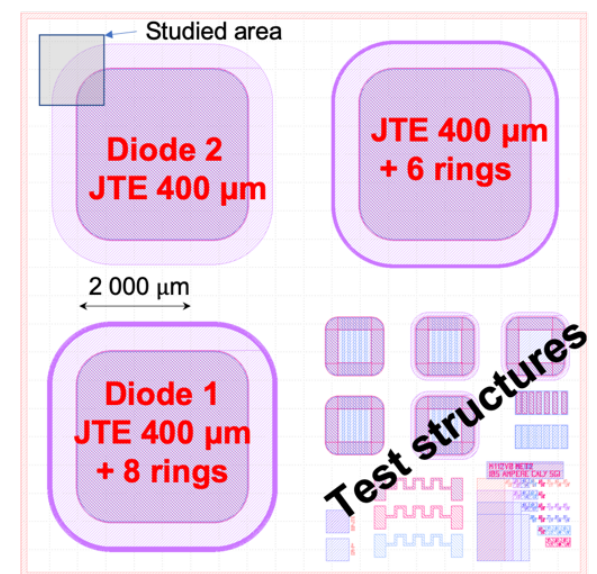

Fig. 3 Elementary cell view of diode mask layout $(1 \mathrm{~cm} * 1 \mathrm{~cm})$

\section{Simulation set-up}

The diode structure design was concurrently simulated thanks to Synopsys Sentaurus TCAD package [9]. The simulator supports all the WBG materials currently used for commercial devices. It does also support custom physical parameters that may be used to feed the available models (Arora, SRH). Based on previous study on the absorption coefficients of UV light in $4 \mathrm{H}-\mathrm{SiC}$ [10], the OBIC and micro-OBIC setup was simulated. The schematic $2 \mathrm{D}$ cross-section of the simulated device is shown in Fig. 4. 


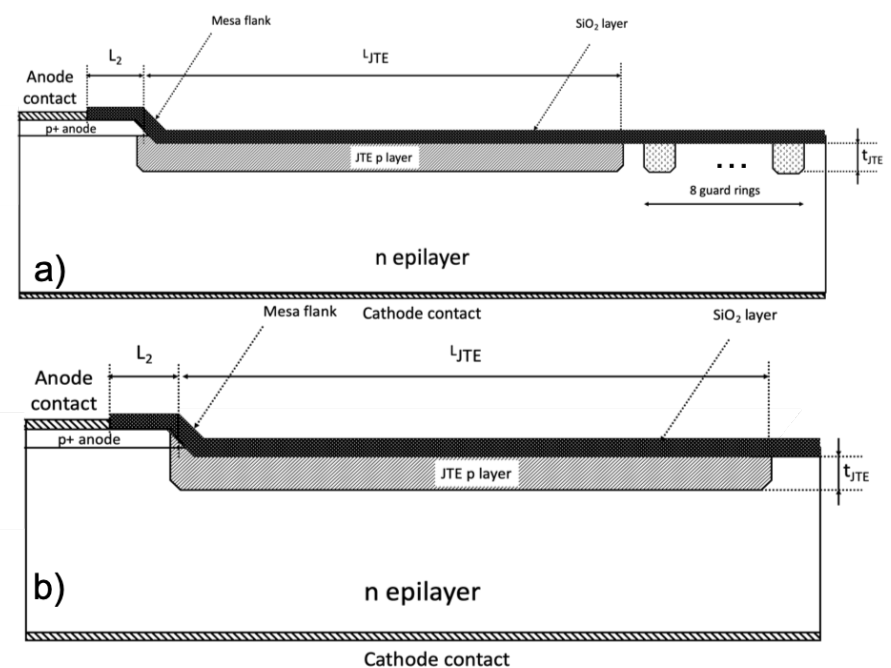

Fig. 4 2D Diode schematic cross-section (a) Mesa JTE diode +8 rings (TCAD diode 1) (b) Mesa JTE diode (TCAD diode 2).

Except the 8 guard rings of the diode 1, both structures share the same features. The $\mathrm{n}$ epilayer is doped at $7.10^{14} \mathrm{~cm}^{-3}$. Its thickness which should be above $100 \mu \mathrm{m}$ has been cut down to $10 \mu \mathrm{m}$ since it will be sufficient for reverse voltage up to $200 \mathrm{~V}$. A JTE layer is formed on the top of the $\mathrm{n}$ epilayer. It is fully covered by a $\mathrm{SiO}_{2}$ layer. The anode sits on the top of a heavily doped $\mathrm{p}+$ region. The distance between the JTE inner edge and the anode contact one is named $\mathrm{L}_{2}$. In order to limit the computing time, only the interesting part of the structure is modelled. The guard rings are properly spaced and their depth is equal to the JTE thickness. The laser beam $(349 \mathrm{~nm})$, located above the structure is defined by a gaussian profile. Its width is adjustable at will. In any case, the beam moves at a constant step of $0.5 \mu \mathrm{m}$. The origin axis is set at the left edge of the structure. The optical generation inside the structure is computed thanks to raytracing. The current obtained across the reverse-biased structure is the simulated OBIC current.

\section{Results and discussion}

\section{Micro-OBIC mapping versus optical image}

$2 \mathrm{D}(\mathrm{X}, \mathrm{Y})$ mapping of the micro-OBIC signal were realized on diode 2 at $0 \mathrm{~V}$ bias. This same diode was observed with an optical microscope. Fig. 5a) and b). compares the optical image and the microOBIC signal of upper left rounded corner the diode. The studied area is represented by a square in Fig. 3.

There is no OBIC signal when the laser beam scans the metallic anode, indeed the anode reflects all the UV light. When the laser beam hits the $\mathrm{p}^{+} / \mathrm{MESA}$ region, the micro-OBIC signal reaches a peak (around $120 \mathrm{nA}$ ), then it decreases to a lower value to stay almost constant (around $30 \mathrm{nA}$ ) in the JTE region. Once it approaches the outer edge of the JTE, the micro-OBIC signal decreases slowly to zero. The optical image shows clearly a first "black ring", around $24 \mu \mathrm{m}$ thick, along the anode metallization. Micro-Raman analysis was performed on this first "black ring" and shows that this black layer is not $\mathrm{SiC}$. This layer can be either a mask or a secondary passivation (polyimide) residues. Further chemical and structural investigations such as SEM/EDX (scanning electron microscopy with energy dispersive X ray spectroscopy) or TOF-SIMS (time of flight second ion mass spectroscopy) are needed to identify this "black layer". It seems that this layer absorbs the visible light and may absorb the UV-light too. If it is the case, an important part of the diode $\mathrm{p}^{+}$will be optically hidden and then OBIC signal would not be observable in this hidden area. A second thinner "black ring" can be observed at $50 \mu \mathrm{m}$ from the anode and is probably due to the mesa edge. 

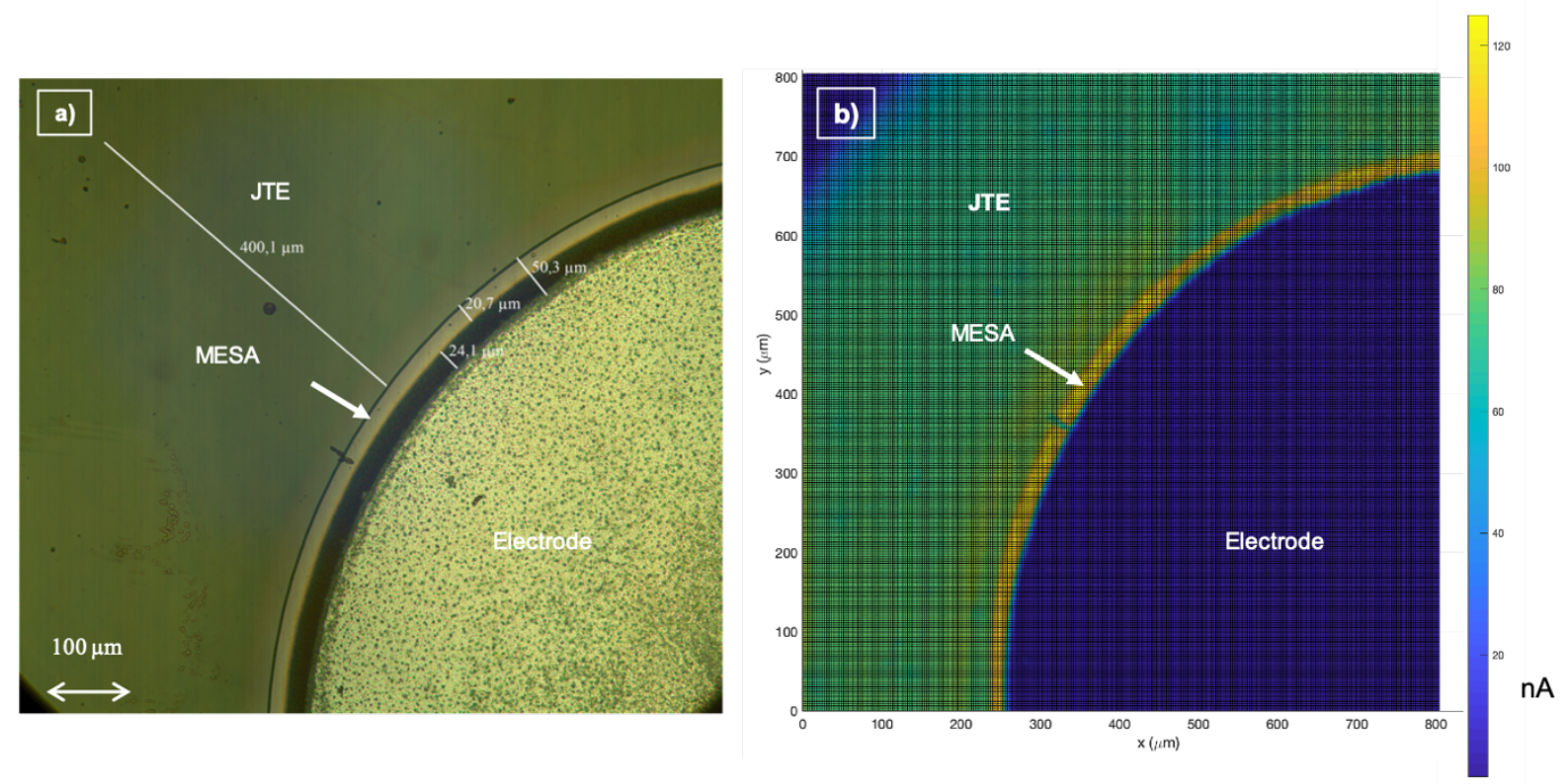

Fig. 5a) (left) Optical image of the diode (top view), microscope objective x50. Fig. 5b) (right) micro-OBIC 2D-scan of the SiC HV PiN diode at OV bias.

Fig. 6a) and $\mathrm{b}$ ) represent a $\left(\mathrm{X}, \mathrm{Y}, \mathrm{I}_{\mathrm{OBIC}}(\mathrm{nA})\right)$ representation in false colors of the OBIC signal with a $0 \mathrm{~V}$ bias (Fig. 6.a)) and at $100 \mathrm{~V}$ reverse bias (Fig. 6b)). The $100 \mathrm{~V}$ reverse bias induced an increase of the OBIC maximum from $120 \mathrm{nA}$ to $200 \mathrm{nA}$ and an increase of the OBIC signal in the JTE region from $30 \mathrm{nA}$ to $130 \mathrm{nA}$. Moreover, the microscopic spatial resolution of the micro-OBIC testbench highlights very small features in the $\mathrm{p}^{+} / \mathrm{MESA}$ region. At $100 \mathrm{~V}$ reverse bias, some defaults in the JTE region are visible. These electrical defaults could be inhomogeneity in the JTE doping concentration. Estimation of the local SiC doping concentration by complementary technics such as micro-Raman spectroscopy could be interesting to realize.
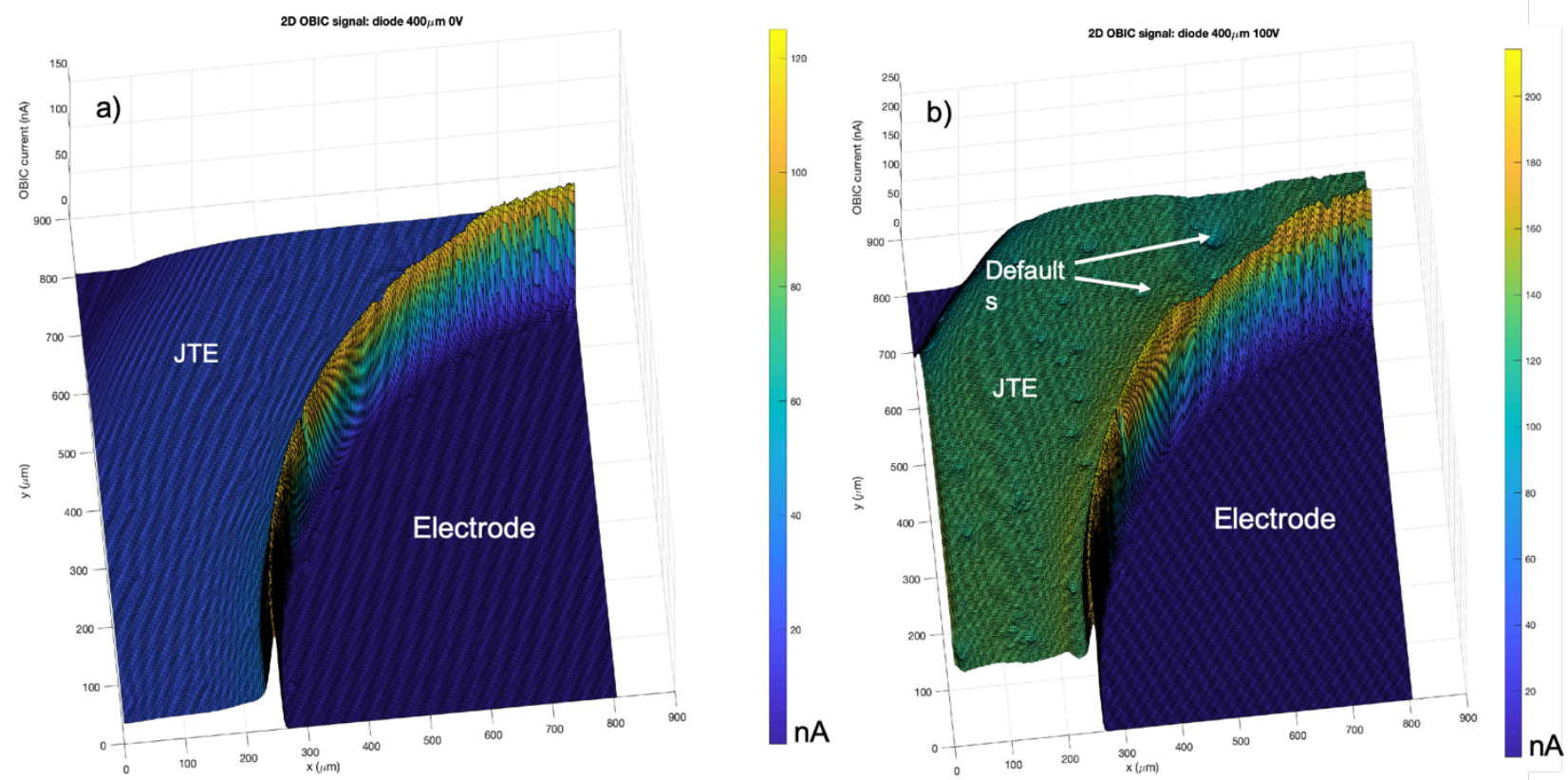

Fig. 6a) (left) Micro-OBIC 2D-scan of the SiC HV PiN diode biased at 0V. Fig.6b) (right) Micro-OBIC 2Dscan of the SiC HV PiN diode reverse biased at 100V. 


\section{Comparison between experimental OBIC and experimental micro-OBIC}

$\mathrm{X}$-line scans were performed with a former OBIC testbench at various reverse voltage on diodes 1 and 2. This former OBIC testbench has been already described in [11] and related measurements published in [5]. X-line scans were performed on diodes 1 and 2 at $0 \mathrm{~V}$ with the new micro-OBIC testbench. Fig. 7a) shows the comparison of the normalized OBIC (in black) and the normalized micro-OBIC (in red) signal for the diode 1. Fig. 7b) shows the comparison between the OBIC (in black) and the micro-OBIC (in red) signal for the same diode 2. The approximative position of the anode and peripheral protections (mesa, JTE, rings) are represented in Fig. 7. The OBIC signals were normalized to have 1 a.u (arbitrary unit) in the JTE region. Fig.7 a) and b) show an important increase of the maximum peak intensity with this new micro-OBIC set-up compared to the OBIC set-up. This observation can be explained by the increase of the spatial resolution.
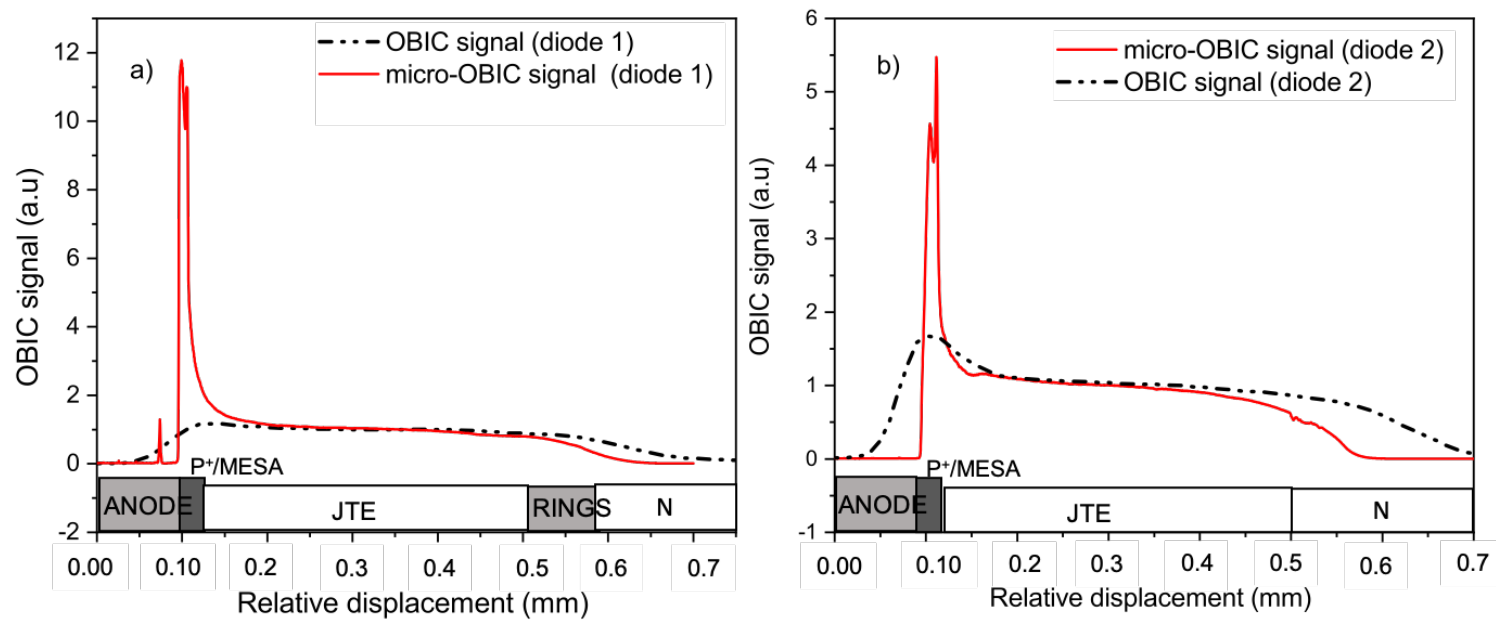

Fig. 7a) (left) OBIC (black dashed) and micro-OBIC (red) X-line scan performed on the 8 JTE rings diode at $0 \mathrm{~V}$. Fig. 7b) (right) OBIC (black dashed) and micro-OBIC (red) X-line scan performed on the no JTE ring diode at $0 \mathrm{~V}$.

In order to estimate experimentally the spatial resolution of the micro-OBIC test bench, we used the experimental procedure described in [2]. For that, we calculated the OBIC and micro-OBIC derivatives in the anode $/ \mathrm{p}^{+}$boundary (i.e when an OBIC signal starts appearing), as shown in Fig. 8. As explained in ref [2], the fullwidth at half maximum (FWHM) of the derivative can well approximate the spatial resolution of the OBIC device. With this method, we estimated the spatial resolution of the former OBIC testbench around $60 \mu \mathrm{m}$. The spatial resolution of the new microOBIC testbench is then $1 \mu \mathrm{m}$ as estimated theoretically. 

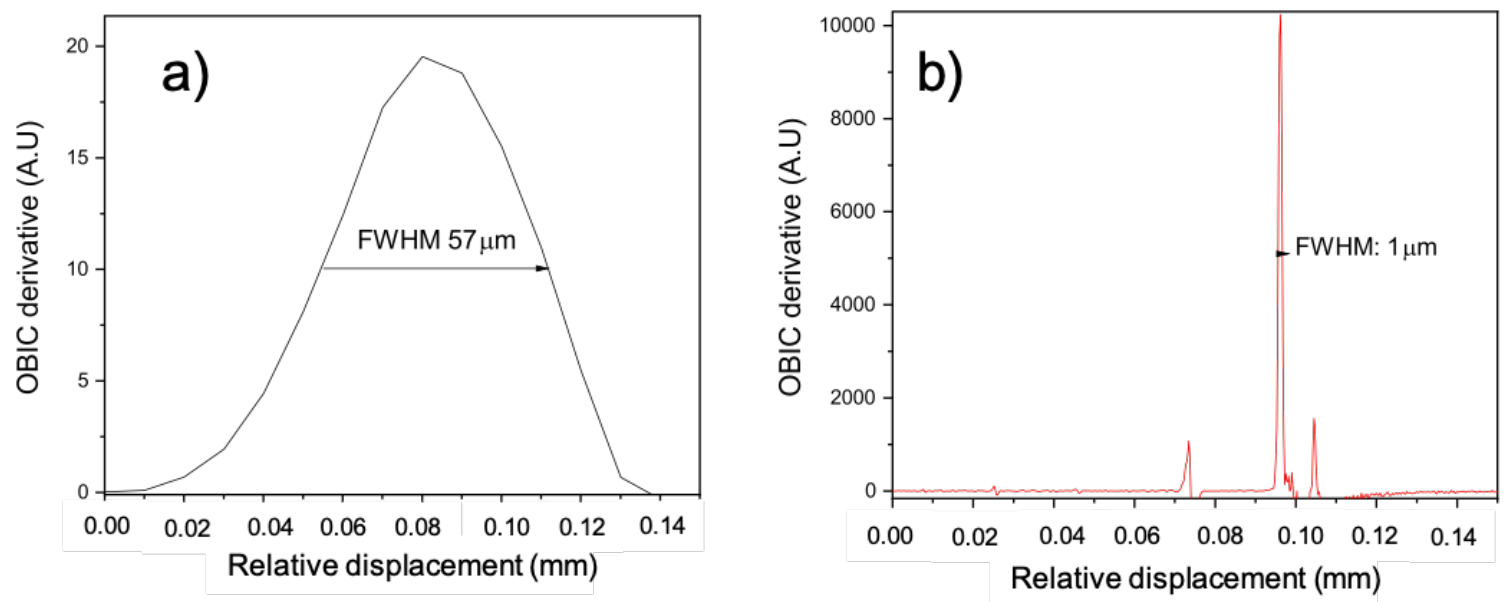

Fig. 8a) (left) Derivative of the diode 1 OBIC signal (black), Fig. 8b) (right) derivative of the diode 1 microOBIC signal (red)

\section{Comparison between experimental and simulated micro-OBIC study of the $\mathrm{p}^{+} / \mathrm{MESA}$ region}

Interestingly the use of well-focused laser beam shows smaller features in the $\mathrm{p}^{+} / \mathrm{MESA}$ region, which were invisible with the previous test bench because of its low spatial resolution. Fig. 9a) is a zoomin of the Fig. 7a) in this $\mathrm{p}^{+} / \mathrm{MESA}$ region. In Fig. 7a) when the micro-OBIC signal appears (relative displacement around $0.1 \mathrm{~mm}$ ), one can observe a sharp current peak with at least two main contributions. To better understand these contributions, we performed some micro-OBIC simulations. Fig. 9b) shows the OBIC current profile obtained from TCAD simulations for a beam width of $1 \mu \mathrm{m}$. In order to be the closest possible to the fabricated diode structure, $\mathrm{L}_{2}$ was set to $7 \mu \mathrm{m}$, which is exactly the part of $\mathrm{p}^{+}$optically visible in Fig. 5a). The simulated beam exposes several features that would merit to be described. First, the left part, from 0 to $22 \mu \mathrm{m}$ on the $\mathrm{x}$ axis, shows no current as expected because the whole beam is reflected. The $7 \mu \mathrm{m}$ wide constant signal $\left(\mathrm{L}_{2}\right.$, from $22 \mu \mathrm{m}$ to 29 $\mu \mathrm{m})$ comes from the $\mathrm{p}^{+}$region exposed to the beam where the SCR stays constant. The profile shows 2 peaks located respectively at 30 and $31 \mu \mathrm{m}$. These peaks are precisely the edges of the MESA. An electric field crowding is responsible for such a profile. As the laser moves towards the outer area of the device, the simulated OBIC current remains constant as it directly mirrors the constant SCR that spreads along the JTE [5].

Fig. 9a) and Fig. 9b) correspond respectively to the experimental and the simulated micro-OBIC signal of diode 1 in the $\mathrm{p}+/ \mathrm{MESA}$ region and have exactly the same scale. Thanks to the simulation results, we can reasonably assume that the MESA edges are responsible for some of the contributions to the OBIC signal experimentally measured.

It should be noted that the simulated OBIC signal does not match completely yet with the experimental one. Firstly, we do not experimentally observe the constant OBIC signal in the $p+$ area. It may be related to the presence of the "black" ring observed in Fig. 5a). Secondly the amplitudes of the OBIC peaks don't match either as the device area is not strictly the same and some other technological uncertainties on samples that must be confirmed. 

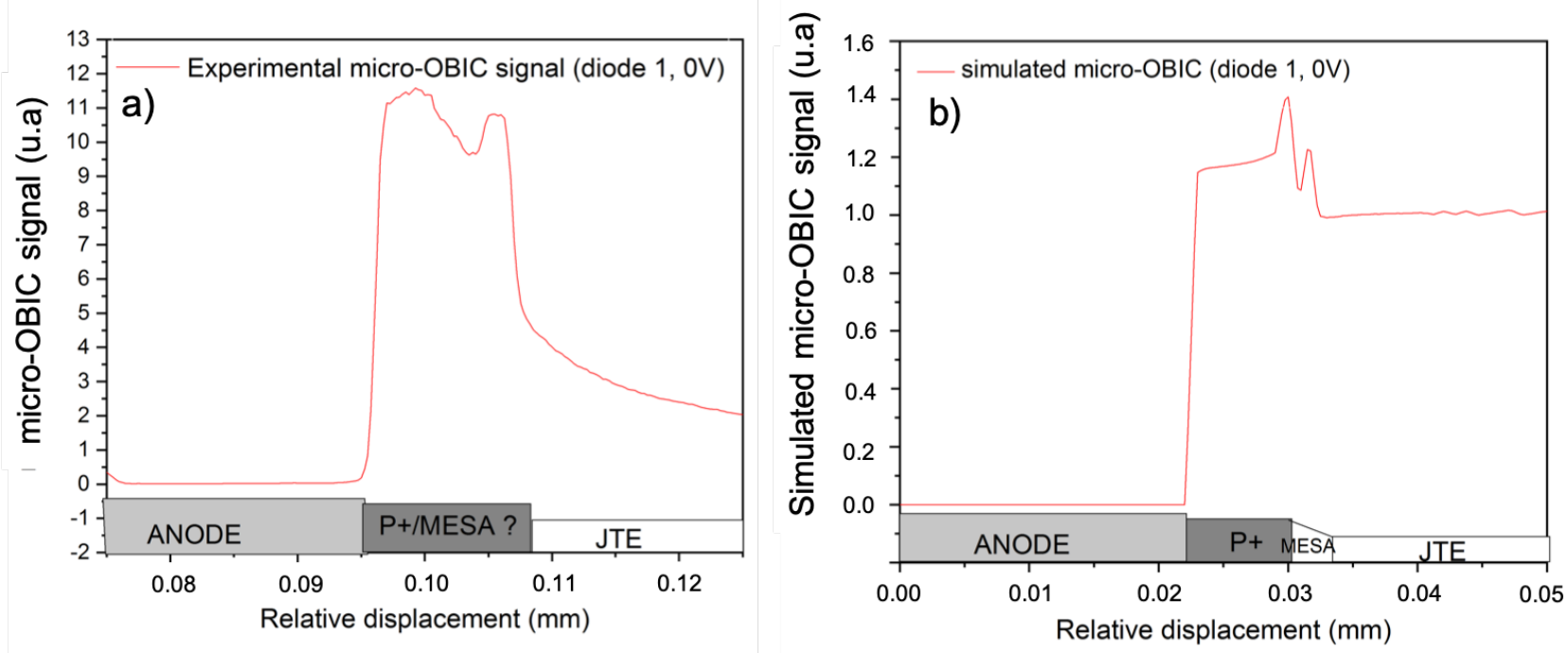

Fig. 9a) (left) Experimental micro-OBIC signal (diode 1, 0V) in the anode/diode border (zoom of Fig. 6b)), Fig. 9b) (right) Simulated micro-OBIC (red) and OBIC (black) signals in the same area (diode 2, 0V).

\section{Comparison between experimental and simulated micro-OBIC study of the JTE outer edge}

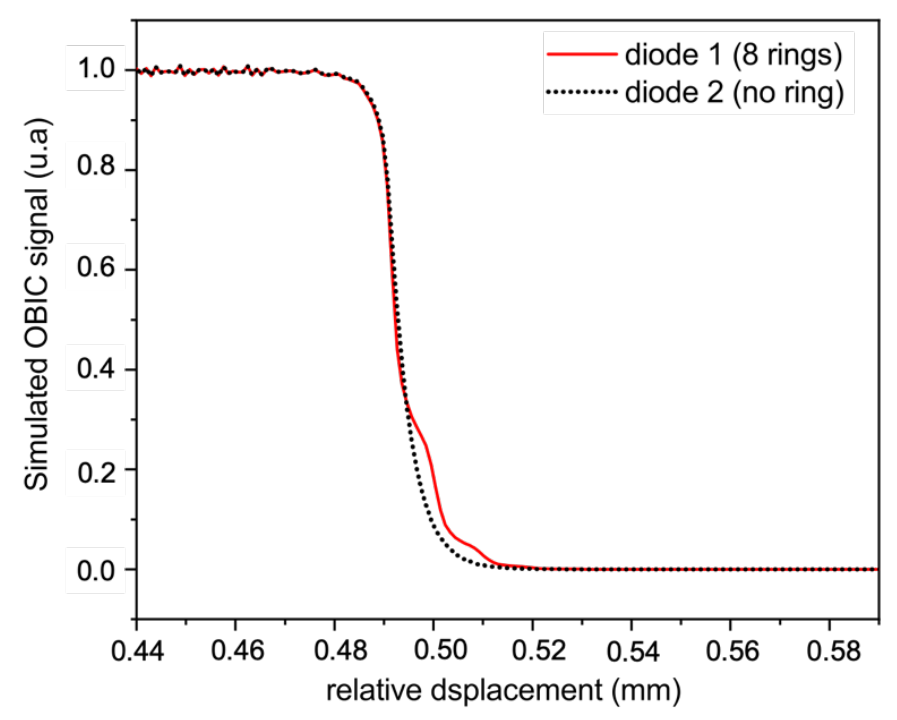

Fig. 10 Simulated micro-OBIC signal in the JTE outer edge of diode 1 (with 8 rings) and diode 2 (without ring) at $0 \mathrm{~V}$.

Fig. 7a) and Fig. 7b) showed that the diode 1 and the diode 2 present some differences in their experimental micro-OBIC signal at the JTE outer edge for $0 \mathrm{~V}$ bias. Indeed, the experimental microOBIC signal of diode 1 (Fig. 7a)) is null for a relative displacement around $0.65 \mathrm{~mm}$ (i.e around 550 $\mu \mathrm{m}$ from the anode edge). Meanwhile, the experimental micro-OBIC signal of diode 2 (Fig. $7 \mathrm{~b}$ )) is null for a smaller relative displacement around $0.58 \mathrm{~mm}$ (i.e around $480 \mu \mathrm{m}$ from the anode edge). As the OBIC signal only exists in presence of a space charge region (SCR), these experimental results mean that there is a further lateral extension of the SCR in diode 1 than in diode 2 . This further lateral extension is around $80 \mu \mathrm{m}$, which is close to the total length occupied by the 8 guard rings.

In order to understand, if these experimental observations can be explained by the presence of the 8 guard rings, we performed some micro-OBIC simulations in the JTE outer edge region. Fig. 10 shows the simulated OBIC in this precise area for diode 1 (with rings) and diode 2 (without rings), the latter 
having ripples and a slight bigger extension of around $3 \mu \mathrm{m}$. Those slight ripples are attributed to a partial depletion of the first guard ring. However, this slight extension of $3 \mu \mathrm{m}$ is far smaller than the one observed experimentally (around $80 \mu \mathrm{m}$ ). Our experimental measurements (see Fig. 7a) and Fig. $7 b$ ) seem to show that the 8 guard rings have been merged together during fabrication and act more like a longer JTE of $80 \mu \mathrm{m}$.

\section{Summary}

We designed a new custom test-bench called micro-OBIC. This test-bench has a well improved spatial resolution compared to the previous OBIC test-bench. We estimated theoretically and experimentally the spatial resolution of the micro-OBIC to be around $1 \mu \mathrm{m}$. A better resolution is interesting to characterize peripheral protections, such as JTE rings or MESA, whose lateral extension is of the order of magnitude of the micron. Our Micro-OBIC experiment and simulations showed that micro-OBIC can be used to characterize the MESA and JTE regions. For example, our micro-OBIC and optical study highlighted some defaults (i.e. "black" rings, JTE inhomogeneities, ...).

Even if JTE rings are not directly observable in these experiments, we observed a further lateral extension of the space charge region (SCR), interpreted as a "merge" of the 8 guard rings to create a longer JTE. It would be then interesting to complete this study by measurements at higher reverse bias.

In order to better understand the defaults, highlighted by our micro-OBIC results, it would be interesting to study these same diodes by other complementary characterization methods such as micro-Raman spectroscopy, SEM/EDX and TOF-SIMS.

\section{Acknowledgements}

The authors want to acknowledge SuperGrid Institute for providing the HV devices and CALY technologies and IBS for the device realization.

\section{References}

[1] T. Kimoto and J.A. Cooper, Fundamentals of Silicon Carbide Technology, Wiley, Sing., (2014).

[2] R. Stengl, High-voltage planar junctions investigated by the OBIC method, IEEE Transactions on electron devices, 34 (1987) 911-919.

[3] H. Hamad, D. Planson, C. Raynaud, P. Bevilacqua, OBIC technique applied to wide bandgap semiconductors from $100 \mathrm{~K}$ up to $450 \mathrm{~K}$, Semicond. Sci. Technol. 32 (2017) 054001.

[4] C. Raynaud, D. Nguyen, N. Dheilly, D. Tournier, P. Brosselard, M. Lazar and D. Planson, Optical beam induced current measurements: principles and applications to SiC device characterization. phys. stat. sol. (a), 206 (2009) 2273-2283. doi:10.1002/pssa.200825183

[5] D Planson, B Asllani, LV Phung, P Bevilacqua, H Hamad, C Raynaud, Experimental and simulation results of optical beam induced current technique applied to wide bandgap semiconductors, Materials Science in Semiconductor Processing, 94 (2019) 116-127

[6] D.M. Nguyen, R. Huang, L.V. Phung, D. Planson, M. Berthou, P. Godignon, B. Vergne, P. Brosselard, Edge termination design improvements for $10 \mathrm{kV} 4 \mathrm{H}-\mathrm{SiC}$ bipolar diodes Materials Science Forum Vols. 740-742 (2013), 609-612. 
[7] P. Hauer, J. Grand, A. Djorovic, G. R. Willmott, and E. C. Le Ru, Spot Size Engineering in Microscope-Based Laser Spectroscopy, J. Phys. Chem. C, 120, 37 (2016) 21104-21113

[8] B. Vergne, S. Scharnholz, R. Hassdorf, D. Planson, D. Tournier, P. Brosselard Precise automated semiconductor characterization under ambient control (PASHAC) - An advanced test bench for highvoltage semiconductor. ISH - International Symposium on High Voltage Engineering, Pilsen, Czech Republic

[9] Synopsys, (2019 09 19) Sentaurus TCAD, Retrieved from:

https://www.synopsys.com/content/dam/synopsys/silicon/datasheets/sentaurus_ds.pdf

[10] J. Hasegawa, L. Pace, L. V. Phung, M. Hatano and D. Planson, "Simulation-Based Study About the Lifetime and Incident Light Properties Dependence of the Optically Triggered 4H-SiC Thyristors Operation," in IEEE Transactions on Electron Devices, vol. 64, no. 3, March (2017), 1203-1208.doi: 10.1109/TED.2017.2657223

[11] D. Planson, B. Asllani, H. Hamad, M.-L. Locatelli, L. Wei, C. Raynaud, P. Bevilacqua, L.V. Phung Near breakdown voltage optical beam induced current (OBIC) on 4H-SiC bipolar diode Materials Science Forum Vol. 924 (2018) pp 577-580 